\title{
Research Paper \\ Evaluating Age-Friendly City Indicators in Qazvin: Urban Open Spaces, Buildings and Public Places
}

\section{Mojgan Kiaie ${ }^{1}$, Seyedeh Ameneh Motalebi ${ }^{2}$, Monirsadat Mirzadeh ${ }^{3}$, , ${ }^{*}$ Fatemeh Mohammadi $^{2}$}

1. Student Research Committee, School of Nursing and Midwifery, Qazvin University of Medical Sciences, Qazvin, Iran.

2. Social Determinants of Health Research Center, Research Institute for Prevention of Non-Communicable Diseases, Qazvin University of Medical Sciences, Qazvin, Iran 3. Metabolic Diseases Research Center, Research Institute for Prevention of Non-Communicable Diseases, Qazvin University of Medical Sciences, Qazvin, Iran.

\begin{tabular}{|c|c|}
\hline $\begin{array}{l}\text { Use your device to scan } \\
\text { and read the article online }\end{array}$ & Citation Kiaie M, Motalebi S, Mirzadeh M, Mohammadi F. Evaluating Age-Friendly City Indicators in Qazvin: Urban Open Spaces, \\
\hline aringin: & $\begin{array}{l}\text { Buildings and Public Places. The Journal of Qazvin University of Medical Sciences. 2019; 23(5):430-439. https://doi.org/10.32598/ } \\
\text { JQUMS.23.5.430 }\end{array}$ \\
\hline astens & d c/ https://doi.org/10.32598/JQUMS.23.5.430 \\
\hline
\end{tabular}

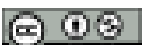

Received: 18 Sep 2018

Accepted: 13 Feb 2019

Available Online: 01 Dec 2019

\section{Keywords:}

Ageing-friendly city,

Ppen space, Build-

ings, Public places

\section{A B STRACT}

Background As the population pyramid in Iran shifts to aging and the influence of environmental conditions on the elderly people, the need to pay attention to the foundations of well-being, security and comfort is felt more than ever.

Objective The present study aimed at evaluating the urban open spaces and public buildings/places of Qazvin city in Iran as indicators of an age-friendly city based on the perception of older people and managers.

Methods This is a descriptive/analytical study conducted in 2017 on 200 older people and 40 urban managers. The older people were selected by a random cluster sampling technique, and managers by a convenience sampling method. Data collection tools were a demographic form and Ageing-Friendly City Questionnaire. Data were analyzed using descriptive statistics and t-test.

Findings The mean age of the elderly people was $68.32 \pm 6.48$ years and for the managers, it was 40.28 \pm 5.94 years. The mean score of public buildings/places was $3.49 \pm 1.06$ reported by the elderly group and $2.64 \pm 0.77$ by the managers. For urban open spaces, it was $3.06 \pm 0.89$ and $2.60 \pm 0.52$, respectively. There was a significant difference between the opinions of two groups regarding the two surveyed indicators $(\mathrm{P}<0.001)$.

Conclusion The city of Qazvin is far from ideal in terms of buildings/public places and open spaces for the elderly. Therefore, urban managers should pay more attention to these indicators and take more effective steps to increase their quality.

\section{Extended Abstract}

\section{Introduction}

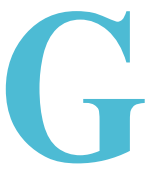

iven the increasing life expectancy and aging population, we need effective policies to promote independence and improve the quality of life of older people. One of these policies is the introduction of the concept of "age-friendly city" $[13,14]$. It is a city that provides active aging for older people by optimizing opportunities for health, participation and security to improve the quality of life, maintain the ability to perform daily living activities and deal with social, cognitive and physical isolation in older people [13]. Given the higher vulnerability of the elderly people to environmental changes affecting urban areas, and considering their disabilities and needs, the appro-

\section{* Corresponding Author:}

Fatemeh Mohammadi

Address: Social Determinants of Health Research Center, Research Institute for Prevention of Non-Communicable Diseases, Qazvin University of Medical Sciences, Qazvin, Iran.

Tel: +98 (912) 1824233

E-Mail: mohammadi1508@gmail.com 
priateness of urban spaces where the elderly need to attend is a major priority of urban adjustment and the creation of a healthy city [16]. Considering the increase of population in cities and the influence of environmental conditions on older people, it is necessary to pay more attention to the issues and principles providing well-being, security and health of this age group so that planners can provide macro-level planning for the upcoming years according to the population pyramid. The present study aimed to evaluate the age-friendly city indicators in Qazvin city, Iran based on the perception of older people and managers in urban open spaces, public buildings/places.

\section{Materials and Methods}

This is descriptive/analytical study conducted in 2017 on 200 older people aged $\geq 60$ years and 40 urban managers in Qazvin city, Iran. The elderly samples were selected by using random cluster sampling technique and the managers by a convenience sampling method. For the elderly samples, inclusion criteria were: age $\geq 60$ years, willingness to participate in the study and ability to communicate verbally. Those with known mental disorders such as Alzheimer's and depression were excluded from the study. For the managers, inclusion criteria were: willingness to participate in the study and experience in the field of aging or urban planning. The data collection tool was a two-part questionnaire including a demographic form and age-friendly city questionnaire designed based on the World Health Organization standards consisted of 14 items assessing indicators of urban open spaces and 5 items assessing indicators of public buildings/places. After explaining the study objectives and methods to the participants and assuring them of the confidentiality of their information, all questionnaires were completed by the researcher through interviewing the participants. The frequency, mean and standard deviation (SD) were used for describing the variables. The independent t-test was used for comparing the indicators for both study groups by determining a cut-off point of 2.5 .

\section{Results}

In this study, the mean age of the elderly group was $68.32 \pm 6.48$ years (ranged 60-84 years) and the mean age of managers was $40.28 \pm 5.94$ years (ranged $30-51$ years). The mean scores of the two variables of urban open spaces and public buildings/places was significantly higher than the cut-off point of 2.5, indicating the existence of a standard limit in Qazvin for these two variables according to the elderly group $(\mathrm{P}<0.001)$, however, the mean scores of managers for these two indices were not significantly higher than 2.5. Regarding urban open spaces, the statement: "the city environment is pleasant and clean" had the highest level of agreement reported by both managers and the elderly group, while the highest level of disagreement was related to the statement: "elderly people have the right of way on a pavement, and the main road junction has special passageways for wheelchairs and trolleys". Regarding public buildings/places, the highest level of agreement was related to the statement: "There are enough toilets in the buildings and public places".

\section{Conclusion}

According to the findings of the present study, Qazvin city was in the standard level in terms of two age-friendly city indicators including public buildings/places and urban open spaces from the perspective of the elderly and urban managers; although, it is far from ideal. In this regard, the results of Zarghani et al. [5] showed that, according to urban managers, the status of open spaces in Mashhad city was favorable, but according to the perceptions of the elderly (Table 1), these spaces are lower than standard level. In the study of Eslami [17] on evaluating the indicators of age-friendly city in Ardabil city, the status of urban open spaces was not acceptable. Findings of Sharqi et al. [16] also showed the unfavorable status of urban open spaces in Tehran city for the elderly people from the point of view of different people such as: physicians, nurses, rehabilitation specialists, designers and urban planners. Therefore, we need proper urban planning to achieve the ideal situation in these two

Table 1. Comparing the perceptions of elderly people and urban managers about urban open spaces and public buildings/places

\begin{tabular}{ccccccc}
\hline Variables & Characteristics & Mean \pm SD & Min & Max & T & Sig. \\
\hline \multirow{2}{*}{ Elderly people } & urban open spaces & $89.0 \pm 06.3$ & 0.01 & 4.39 & 8.28 & 0.00 \\
& public buildings/places & $07.1 \pm 49.3$ & 0.01 & 5.00 & 13.90 & 0.00 \\
Urban managers & urban open spaces & $53.0 \pm 60.2$ & 1.92 & 3.05 & 1.02 & 0.42 \\
& public buildings/places & $77.0 \pm 64.2$ & 1.02 & 4.02 & 1.11 & 0.72 \\
\hline
\end{tabular}


areas. With regard to the urban open spaces, we need to pay more attention to the sidewalks and special passageways for wheelchairs and trolleys, and in terms of public buildings/places, we need to be careful in the construction and installation of elevators, escalators and sanitary facilities, taking into account the age limitations.

\section{Ethical Considerations}

\section{Compliance with ethical guidelines}

Prior to study, after explaining the objectives of the study and assuring them of the confidentiality of the information, a written informed consent was obtained from the participants. This study was approved by the Research Ethics Committee of Qazvin University of Medical Sciences (Code: IR.QUMS.REC.1395.27).

Funding

This article was extracted from a master thesis approved by Mojgan Kiaie in Gerontological nursing approved by Qazvin University of Medical Sciences.

\section{Authors' contributions}

Methodology: Fatemeh Mohammadi, Seyedeh Ameneh Motalebi, Mojgan Kiaie; Data Analysis: Monirsadat Mirzadeh; Resources and Initial Draft Preparation: Fatemeh Mohammadi, Seyedeh Ameneh Motalebi, Mojgan Kiaie; Editing: Fatemeh Mohammadi, Seyedeh Ameneh Motalebi; Project Administration: Fatemeh Mohammadi.

\section{Conflicts of interest}

The authors declared no conflict of interest.

\section{Acknowledgements}

The authors would like to thank the authorities of School of Nursing and Midwifery of Qazvin University of Medical Sciences, and all elderly people and managers participated in the study for their valuable cooperation. 


\title{
ارزيابى شاخصهاى شهر دوستدار سالمند شهر قزوين: فضاهاى باز شهرى، ساختمانها و مكان هاي عمومى ساخمى
}

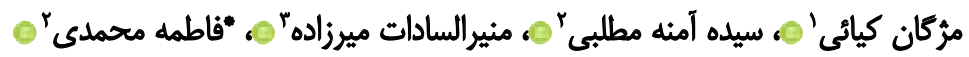

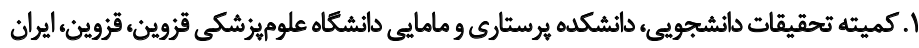

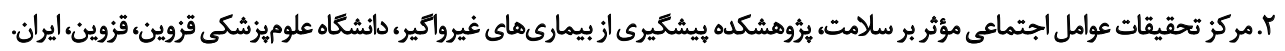

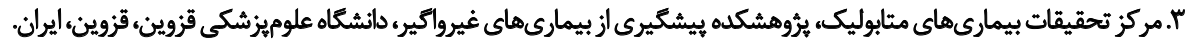

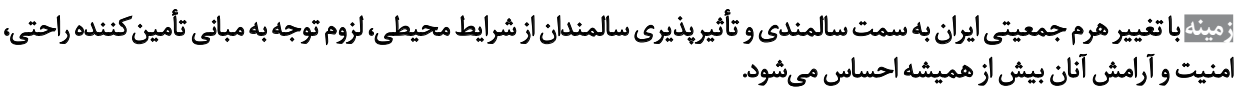

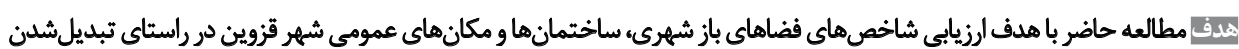

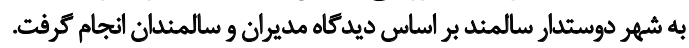

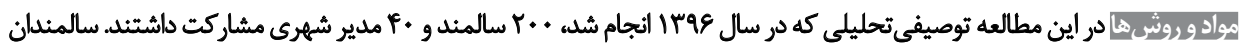

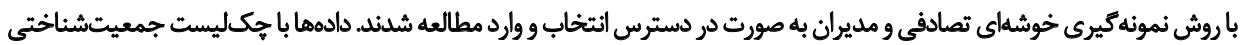

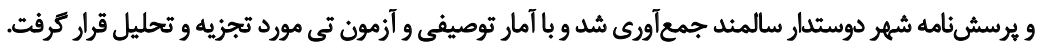

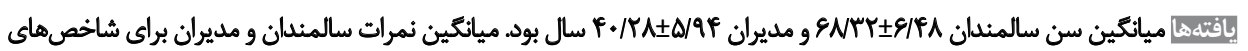

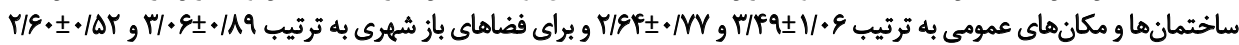

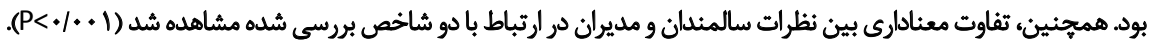

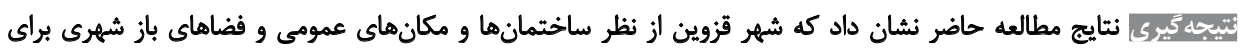

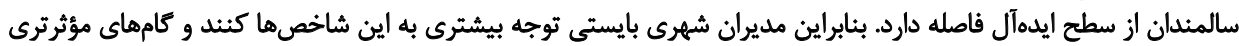
جهت افزايش كيفيت آنها بردارند.

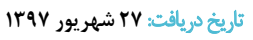

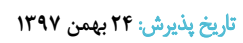

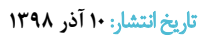

كليدوأوهاها: شُهر دوستدار سالمنده فضاى باز، ساختمانها،

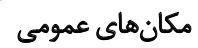

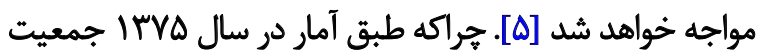

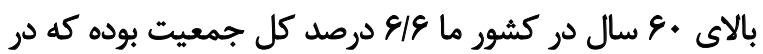

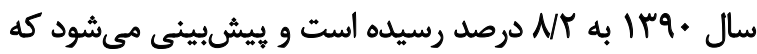

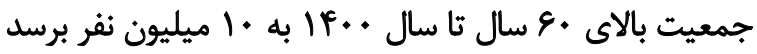

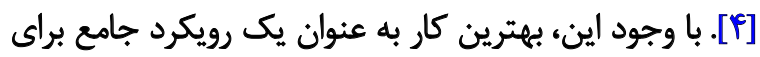

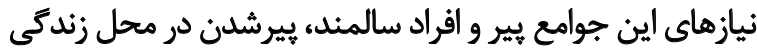

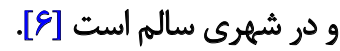

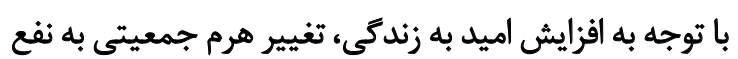

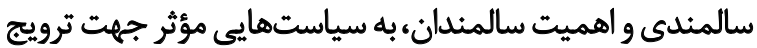

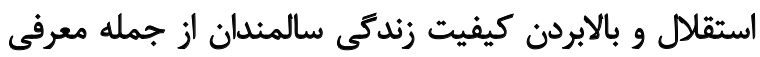

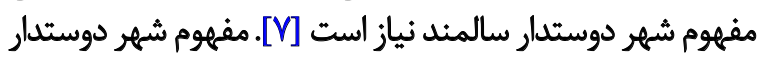

بيشرفتهايي كه در زمينههاى مختلف، بهويرٔه بهبود تغذيه،

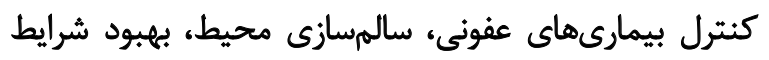

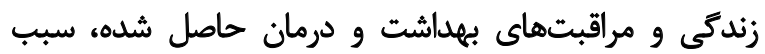

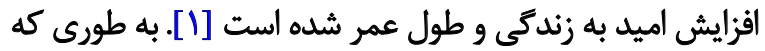

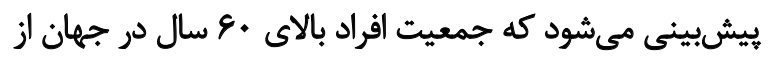

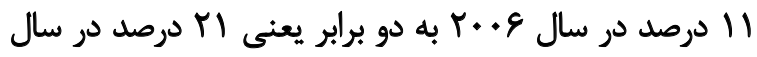

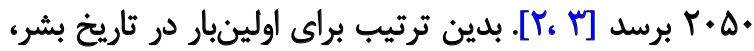

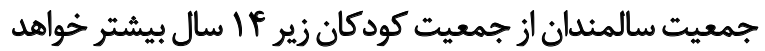

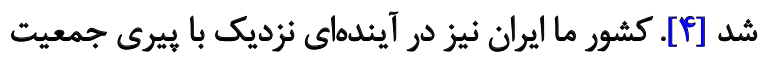

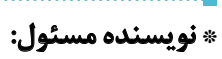

$$
\begin{aligned}
& \text { فاطمه محمدى فوينده }
\end{aligned}
$$

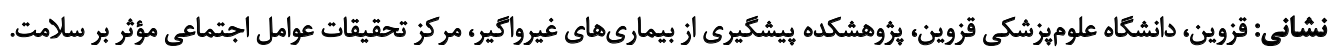

$$
\begin{aligned}
& \text { تلفن: } \\
& \text { رايانامه: mohammadi1508@gmail.com }
\end{aligned}
$$




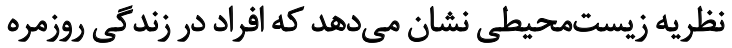

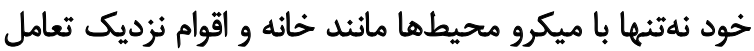

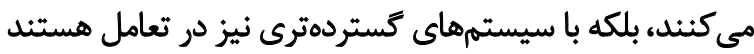

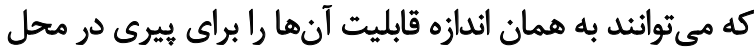

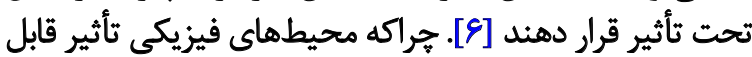

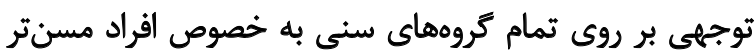

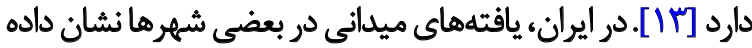

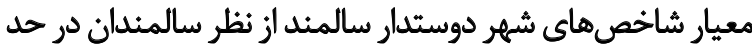

متوسط و حتى ضعيف بوده است [If]

بادرنظركرفتن افزايش شهرنشينى و تأثيريذيرى افر ادراد سالمنداز

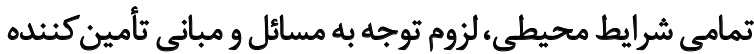

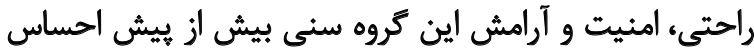

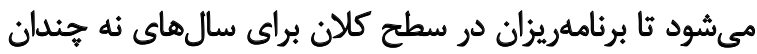

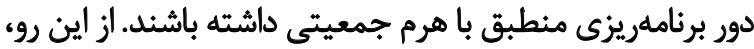

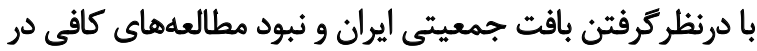

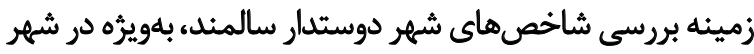

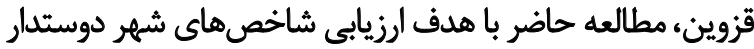

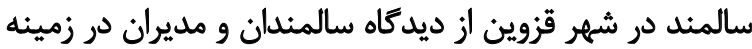
فضاهاى باز شهرى، ساختمانها و مكانهاي عمومى انجام كرفت.

\section{مواد و ووشهها}

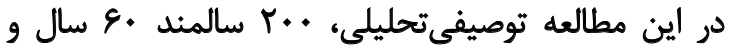

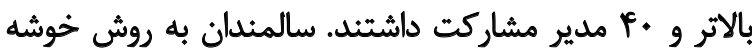

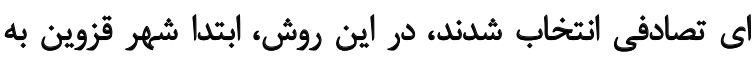

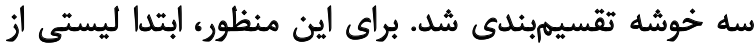

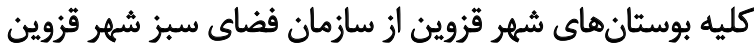

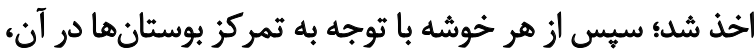
تعدادى بوستان به صورت تصادفى انتخاب شديند.

مسئولين از استاندارى و شهردارى سه منطقه به صورت در

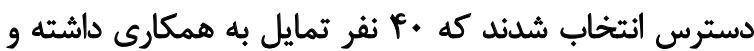

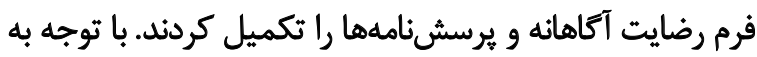

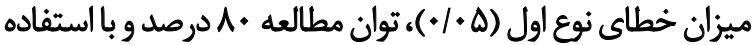

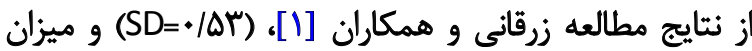

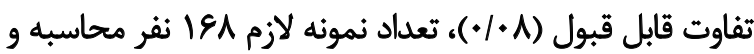

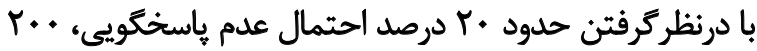
تقفر تعيين شد.

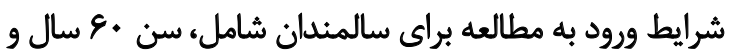

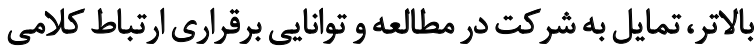

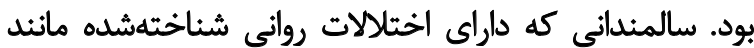

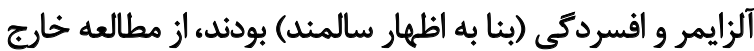

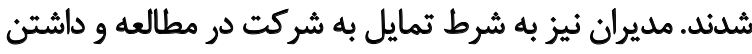

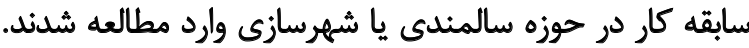

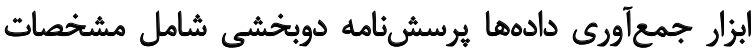

سالمند، دو تمايل اصلى براي بيرى و شهرنشينى را الرائه ميدهد

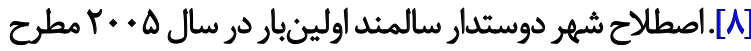

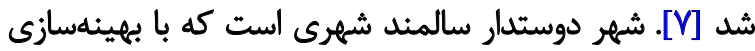

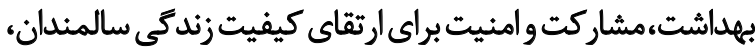

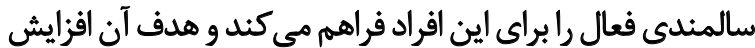

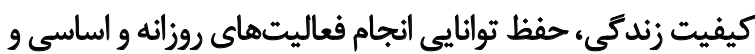

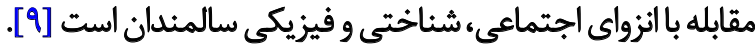

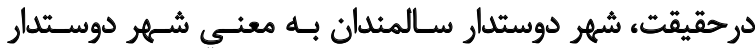

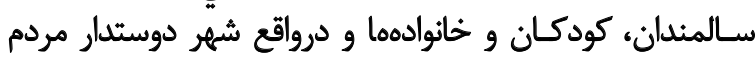

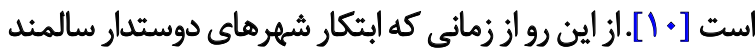

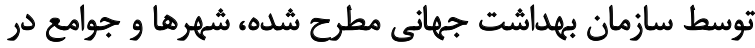

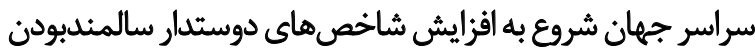

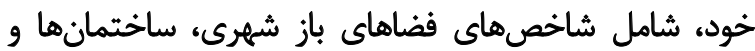

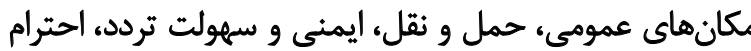

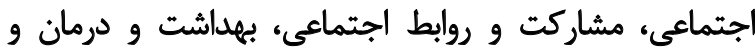

شاخصهاى فرهنكى تفريحى كرداند [ [1].

فضاي باز شهري از ديرباز تاكنون بستر تعاملات اجتماعي بوده

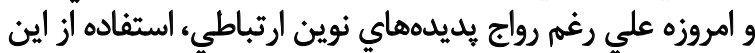

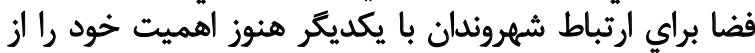

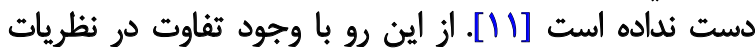

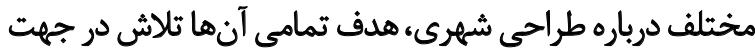

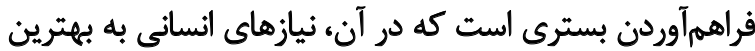

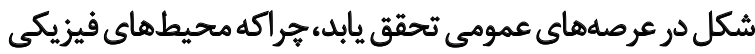

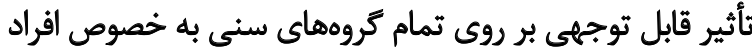

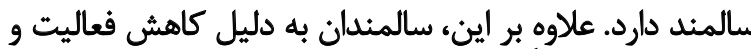

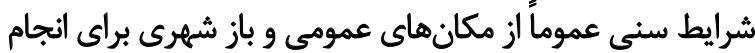

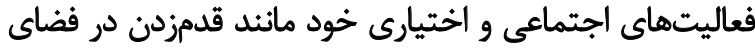

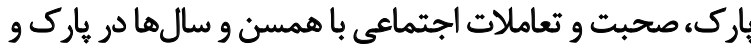
خيابانها استفاده مى كنيند [111].

تحقيقات بسيارى كه به بررسى تأثير ساخت محيط بر روى

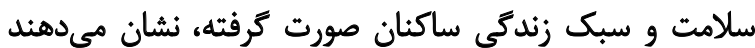

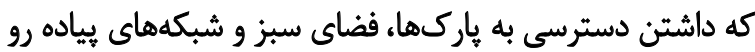

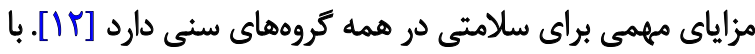
توجه به آسيبيذيري بيشتر سالمندان نسبت به به تغييرات محيطي

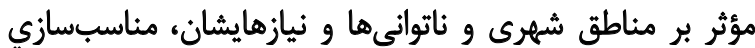

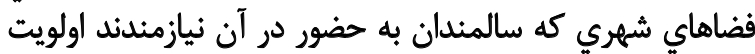

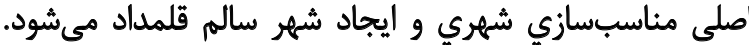

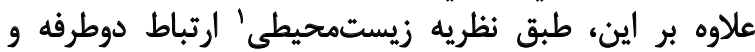

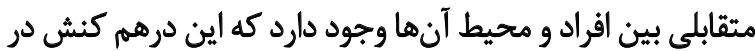

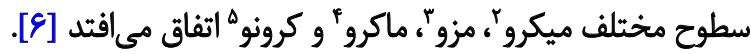

1. Bio-environmental theory

2. Micro

3. Meso

4. Macro

5. Corono 


\section{يافتهانا}

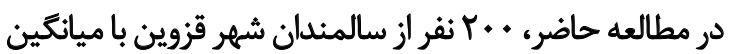

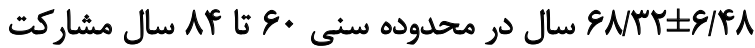
داشتند. ميانكين سن مديران

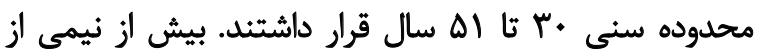

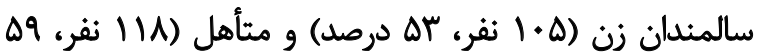

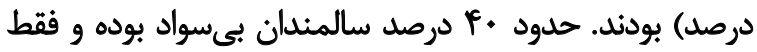

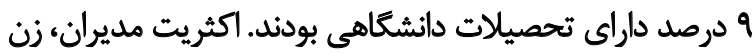

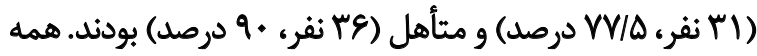
مديران داراى تحصيلات دانشكاهى بودند.

همان طور كه اطلاعات جدول شماره ا نشان مي دهد، ميانكين

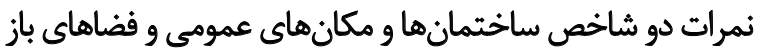

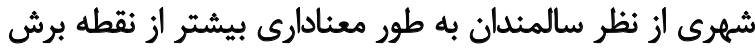

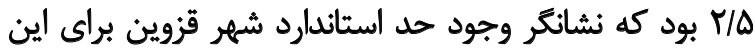

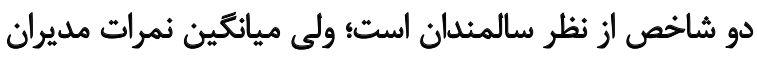

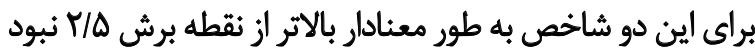

$$
\text { (سطح معنى دارى = ه • (*). }
$$

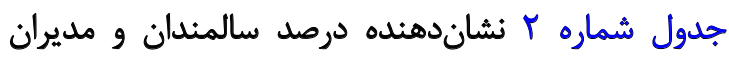

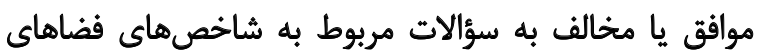

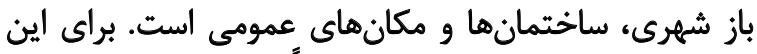

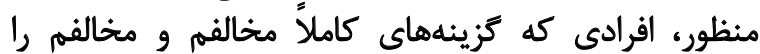

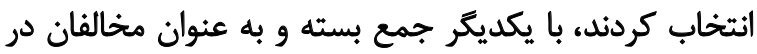

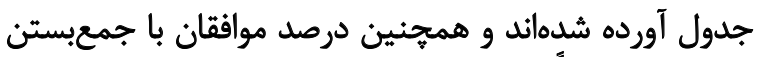

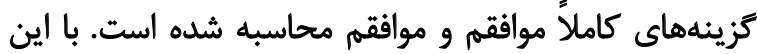

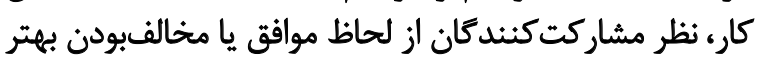

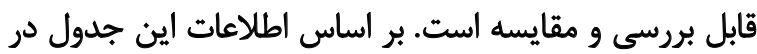

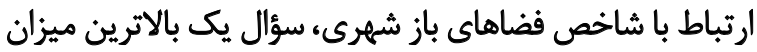

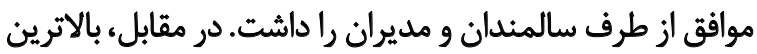

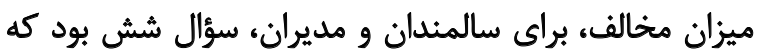

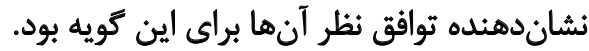

جمعيتشناختى (سن، جنس،وضعيت تأهل و سطح تحصيلات)

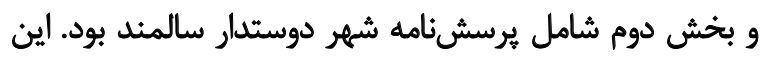

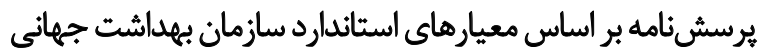

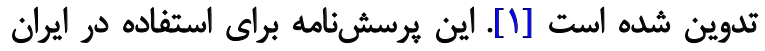

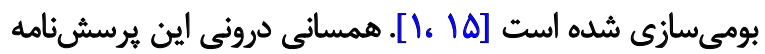

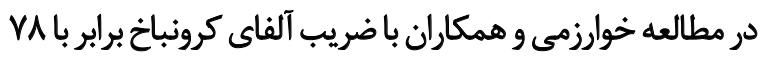

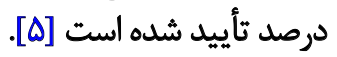

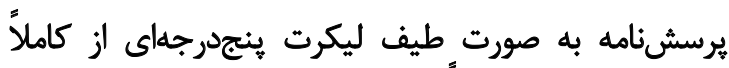

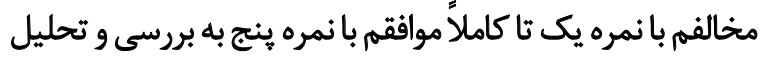

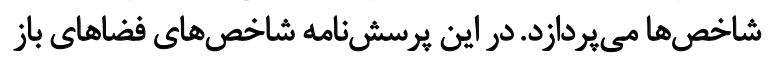

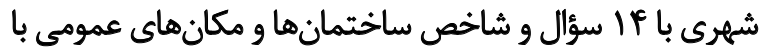

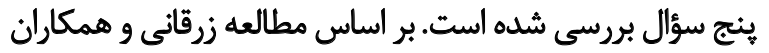

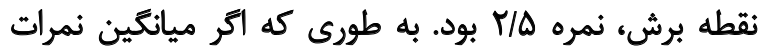

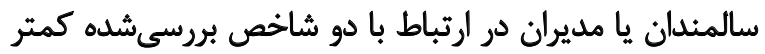

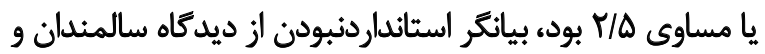

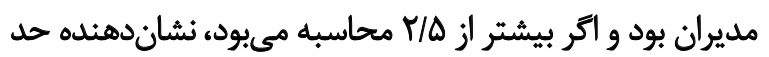

استاندارد اين شاخص بود [1]

يس از اخذ معرفينامه رسمي از معاونت يُروهشى دانشكاه

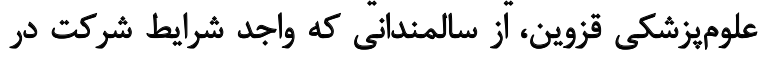

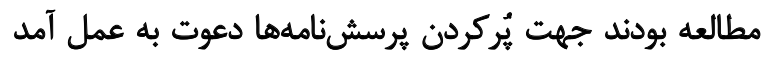

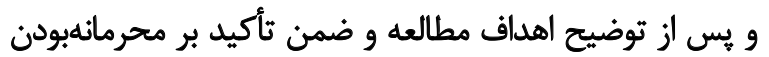

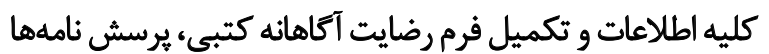

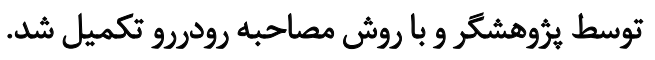

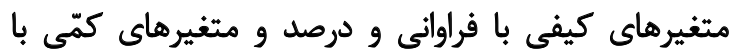

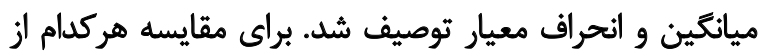

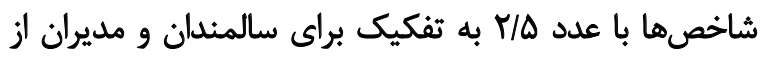

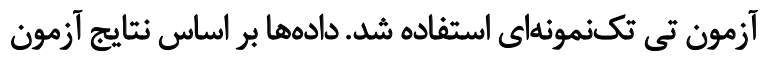

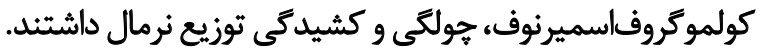

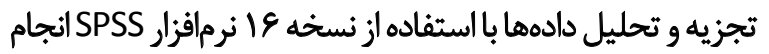

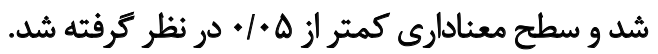

جدول ا. مقايسه ديدكاه سالمندان و مديران در ارتباط با شاخصهاي فضاهاى باز شهرى، ساختمانها و مكانهاى عمومي

\begin{tabular}{|c|c|c|c|c|c|c|}
\hline (أزمون تى تكنى معنىنهاىى) & آماره تى تكـنمونهاى & ماكزيمم & مينيمم & ميانكين Iانحراف معيار & شاخص & شركت كنندكان \\
\hline $100^{\circ}$ & NAT & $r / q r$ & $1 \%$ & $\mathrm{H} / .8 \pm . / 19$ & فُضاهاى باز شهرى & \\
\hline$++^{*}$ & $14 / \div 9$ & $\Delta / \omega$ & $\mathrm{V} \cdot *$ & $r / F q \pm V / * r$ & ساختمانها و وكانهاى & سالمندان \\
\hline.$/ \pi$ & $1 / r$. & $r / \Delta$. & $1 / r q$ & $r / \varepsilon \cdot \pm \cdot / \Delta r$ & فضاهاى باز شهرى & \\
\hline.$/ T V$ & $1 / 11$ & $\varphi / T$. & $V / r$ & $r / E T \pm . W$ & ساختمانها و مكانهاى & مديران \\
\hline
\end{tabular}


جدول Y. درصد باسخ شاخصهاى فضاهاى باز شهرى، ساختمانها و مكانهاى عمومى

\begin{tabular}{|c|c|c|c|c|}
\hline \multicolumn{2}{|c|}{ 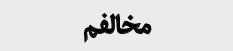 } & \multicolumn{2}{|c|}{ 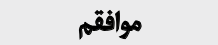 } & \multirow{2}{*}{ فضاهاي باز شهرى } \\
\hline مديران & سالمندان & مديران & سالمندان & \\
\hline$r V / \Delta$ &.$/ \pi$ & $9 \pi / \Delta$ & $\Delta N \Delta$ & ا. محيط شهر دلهذير و تميز است \\
\hline$M / Q$ & $r \Delta / \Delta$ &.$/ 4$ & rNa & r. وضعيت كنترل سر و صداو بوهاى نامطلوب و آلودكى هوا در مكان هاى عمومى بهخوبى اجرا مىشود \\
\hline$V / \Delta$ & $\mathrm{r} / \mathrm{\Delta}$ & $V / \Delta$ & $\Delta F / \Delta$ & ".. به تعداد كافى محل هايع براى استراحت سالمندان تعييه شده است \\
\hline$\Delta r / \Delta$ & $r \cdot / \Delta$ & D & $\Delta r / \Delta$ & F. در فضاهاى سبز مكان هاى مسقف و سايهدار به همراه صندلىهاى راحت و ايمن به تعداد كافى در فواصل منظم وجود دارد \\
\hline$\Delta r / \Delta$ & $\pi / \Delta$ & $m / \Delta$ &.$/ \% \mathrm{r}$ & هـ در ايسكاههاى حمل و نقل عمومى محيط و على الخصوص صندلى هائ راحت، ايمن و به ميزان كافى وجود دارد \\
\hline$\cdot / \Lambda$. & .190 &.$/$ &.$/ T \Delta$ & ؤ در يبادرووها اولويت عبور سالمند رعايت شده است و محل اتصال به جاده اصلى داراى معابر ويزه براى ويلجر و جخ دستى \\
\hline$r / \Delta$ &.$/ 4 \Lambda$ &.$/ 4$. &.$/ \Delta V$ & V. در شهر به ميزان كافى توالت عمومى با امكاتات و بهداشت مناسب وجود دارد \\
\hline$n / a$ &.$/ 49$ & $1 \pi / \Delta$ & 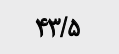 & ه در توالتهاى عمومى، توالت مناسب براى سالمندان تعييه شده است \\
\hline EN/D &.$/ 4 V$ & .1 &.$/ 4$ & ه. خيابانها بدون شيب بوده و با قرارداشتن خطكى هاى عابر يياده نزديك به هم امكان عبور ايمن و مطمئن براى سالمندان \\
\hline$\cdot / V \Delta$ & $\Delta / T V$ &.$/ \pi$ & PNA. & •ا. جراغهاى عابر يياده و سيكنال هاى صوتى و تصويرى مناسب و قابل رؤيت براى سالمندان به منظور عبور سالم و مطمثن \\
\hline$\cdot N$ & r &.$/$ & $\circ / R$ & 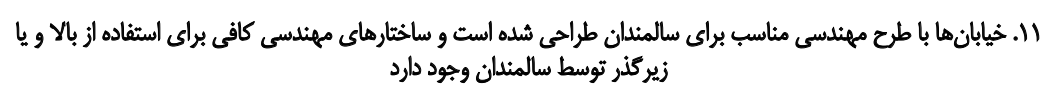 \\
\hline$\pi / \Delta$ &.$/ 4 V$ & $\mathrm{PV} / \mathrm{\Delta}$ &. & rا. زمان جراغهاى سبز براى عبور عابر يياده سالمند و يا با ويلجر بسيار كم و نامناسب است و علاثاثم صوتى و بصرى لازم نيز \\
\hline .18. & $\Delta / \%$ & $\pi / \Delta$ & $\varphi / \Delta$ & سا. محل توقف تاكسى و اتوبوس مثاسب سالمثدان است \\
\hline ET/D & $.1 \Delta 1$ &.$/ R \Delta$ &.$/ \%$ & با. ورودى ياركها براى ويلجر و جرغ دسثى وغيره مناسب است \\
\hline \multicolumn{2}{|c|}{ 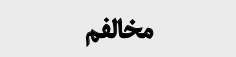 } & \multicolumn{2}{|c|}{ موافقم } & \\
\hline مديران & 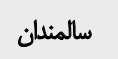 & 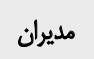 & مالمنلان & 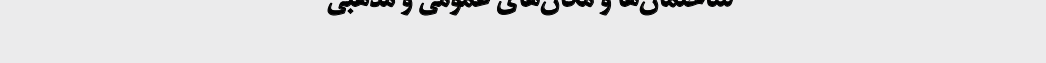 \\
\hline$\Delta / \Delta V$ &.$/ 4 \Delta$ & $\pi / \Delta$ & $\Delta \cdot / \Delta$ & ا. ساختمانهاى عمومى داراى آسائسور، يلهبرقى يا يلههايع با شيب و ارتفاع كم و داراى نرده و سطوح غيرلغزئده هستد \\
\hline$\Delta / \& V$ &.$/ 4 \Lambda$ & $\mathrm{r} / \mathrm{s}$ & $g / / \Delta$ & ك. اطلاعات كافي براى نشاندادن مسير و همجنين علائم هشداردهنده مناسب وجود دارند \\
\hline.$/ \% a$ &.$/ T \Delta$ &.$/ \% \Delta$ & $e x / \Delta$ & ب. در طبقات سائتمان ها و مكانهاى عمومى، توالت به اندازه كافى وجود دارد \\
\hline $9.1+$. & $\pi / \Delta \cdot$ & $+/ \pi \Delta$ & $\Delta V / \Delta$. & F. در طبقات ساختمانها و مكان هاى عمومى، مكان هاى استراحت با صندلىهاى راحت به اندازه كافى وجود دارد \\
\hline$v \Delta / \Delta$. & $\pi / \Delta \cdot$ &..$/ r \Delta$ & $\Delta T / \Delta$. & هـ. در ساختمان ها و مكان هاى عمومى، علاثم مشخص و مناسب دسترسى به امكانات مختلف وجود دارد \\
\hline
\end{tabular}


از ديدكاه سالمندان ساختمانها و مكانهاى عمومى و مذهبى رئي

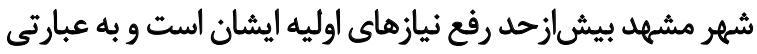

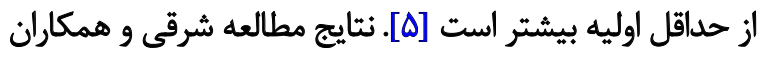

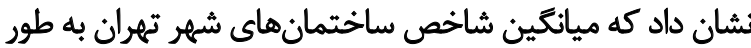

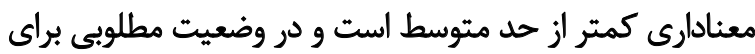

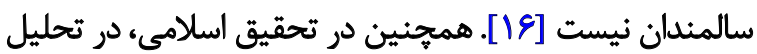

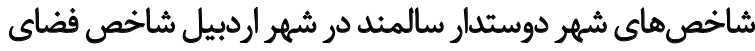

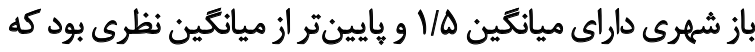

وضعيت قابل قبولى نداشت [IV]

شايد بتوان تفاوت در نتايج را به اين صورت تفسير كرد كه

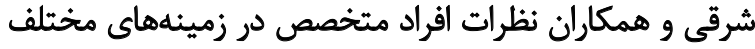

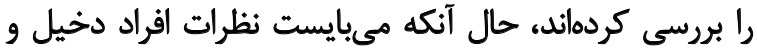

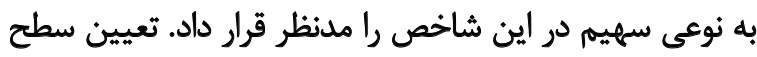

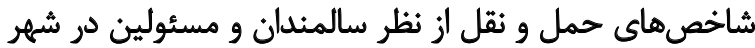

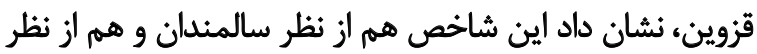

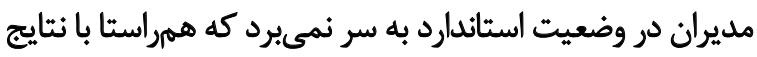

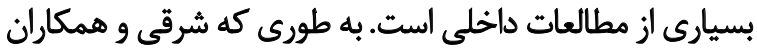

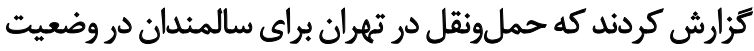

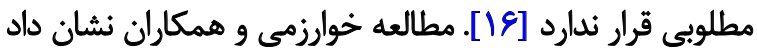

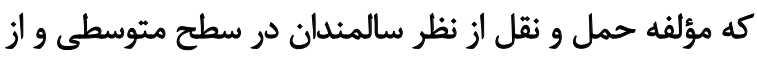

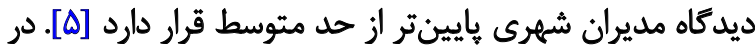

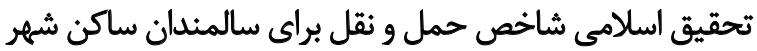

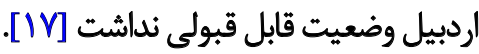

در مطالعه ترقى نيز مشخص شد كه اكثريت سالمندان ساكن

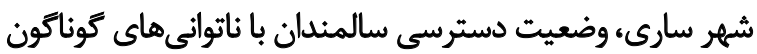

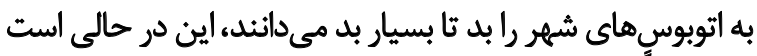

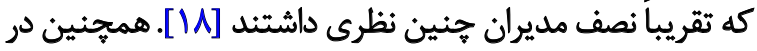

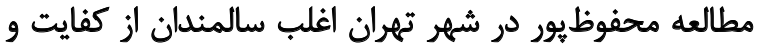

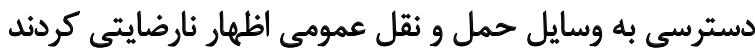

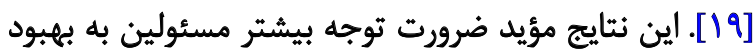
شاخص حمل و نقل براى سالمندان است.

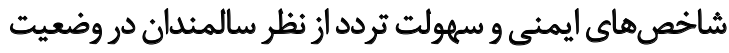

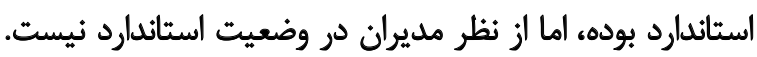

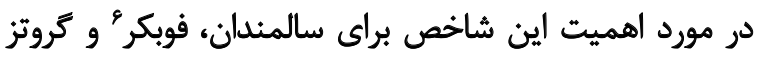

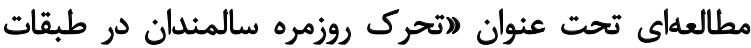

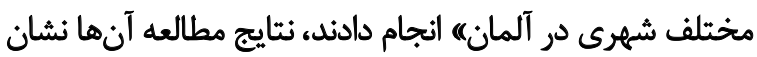

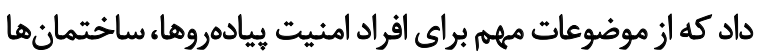

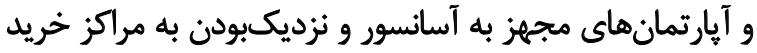

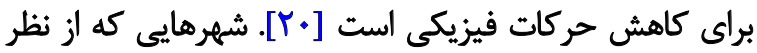

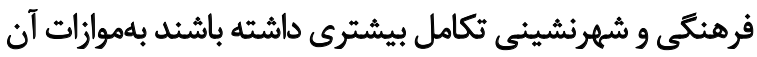

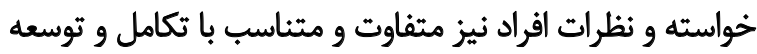

6. Fobker
در ارتباط با درصد ياسخهاى موافق و مخالف به كويههاى

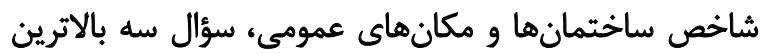

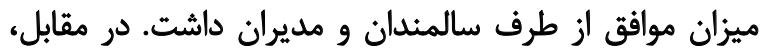
سؤال يك از طرف سالمندان و سؤال دو از نظر مدير مدان مان بان بالاترين

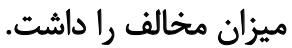

\section{بحث و نتيجنه كَيرى}

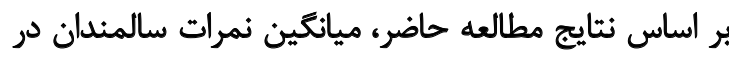

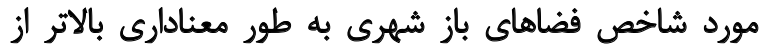

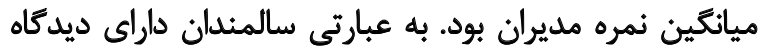

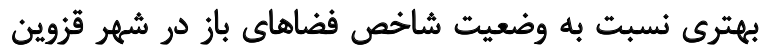

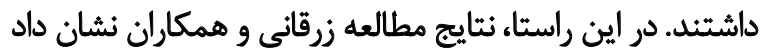

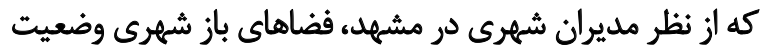

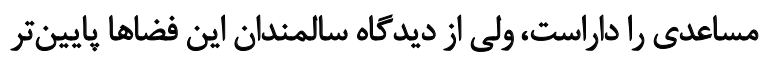

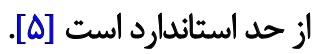

تفاوت در نتايج كزارش سالمندان را مى توان اينكونه تفسير

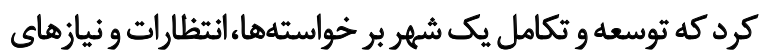

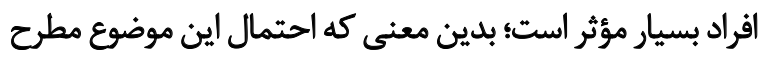

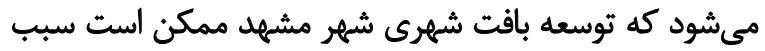

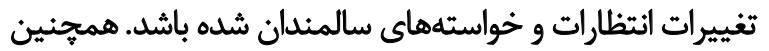

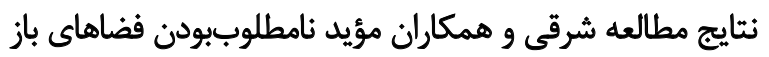

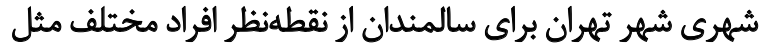

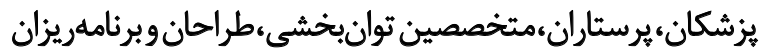

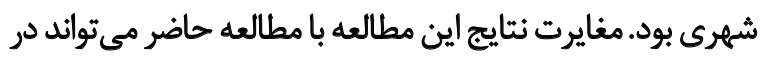

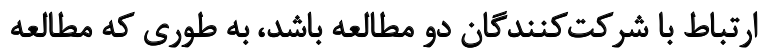

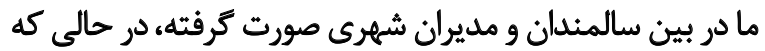

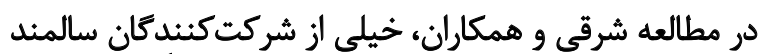

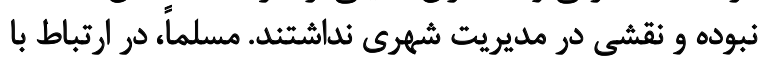

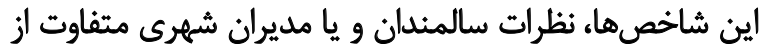

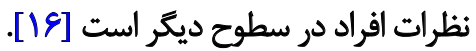

در تحقيق اسلامى، در تحليل شاخصهاى شهر دوستدار

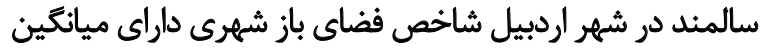

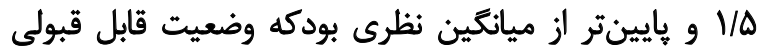

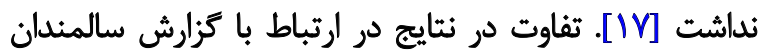

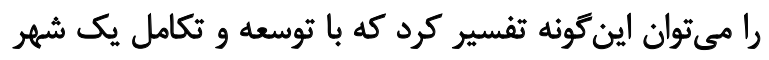

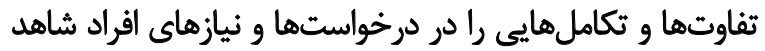

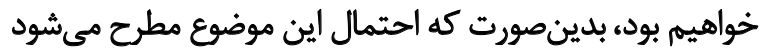

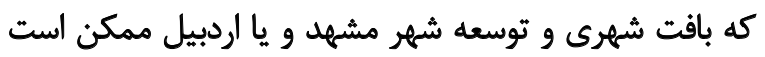
سبب تغييرات انتظارات و خواستههاي سالمندان شده باشيد.

تعيين سطح شاخصهاى ساختمانها و مكانهاى عمومى

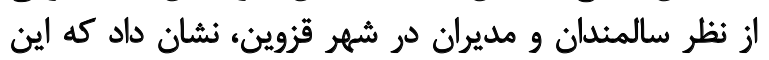

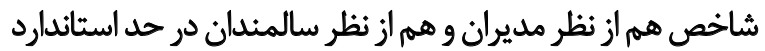

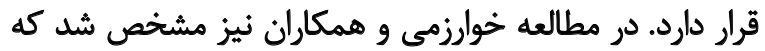


با كد IRQUMS.REC.1395.27 مورد تأييد قرار كرفت. به

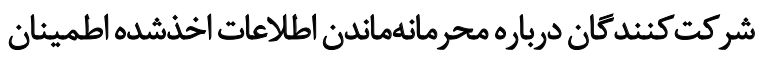

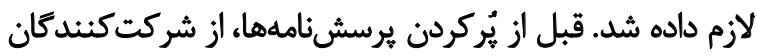
رضايت كتبى كرفته شد.

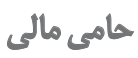

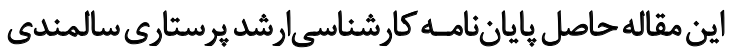

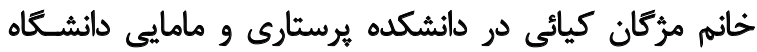

$$
\text { علـوميزشـكى قزوين استي }
$$

$$
\text { مشاركت ثويسند تكان }
$$

روش تشناسي: فاطمه محمدى، سيده آمنه مطلبى و مرّكان

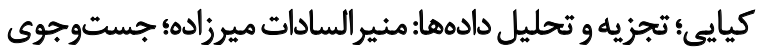

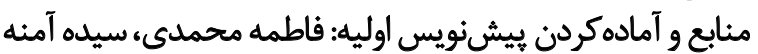

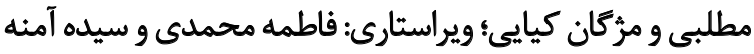
مطلبى؛ مديريت يرورث: فاطمه محمدى

$$
\text { ت تعارض مثافع }
$$

بنا به اظهارنظر نويسندكان، اين مقاله تعارض منافع ندارد.

$$
\text { تشيكر و قبدرواتى }
$$

بدينوسيله از مسئولين محترم دانشكده يرستارى مامايى

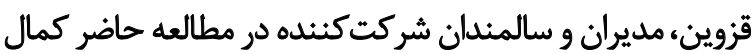

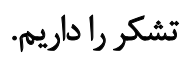

شهرى خواهد بود.در مطالعه سيو و و همكار ان نتايج مطالعه نشان

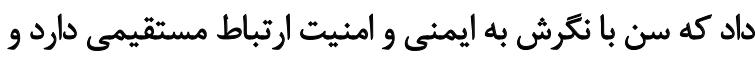

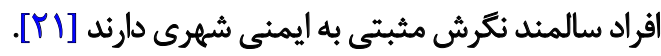
استكهلم در سال rا • r موفق به دريافت جايزه شهر دوستدار

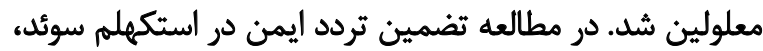

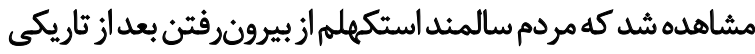

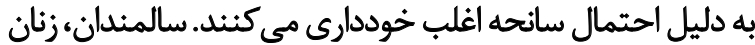

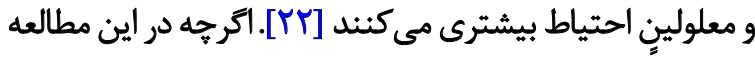

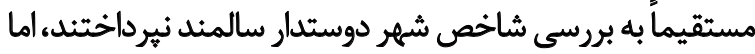

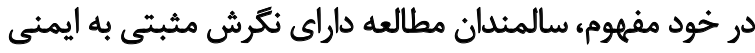
شهرى بودهاند كه همراستا با نتايج مطالعه ماست.

تعيين سطح شاخصهاى بهداشت و درمان از نظر سالمندان

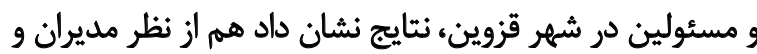

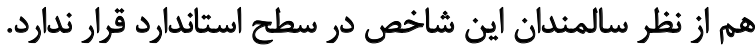

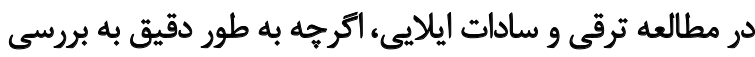

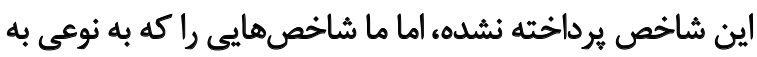

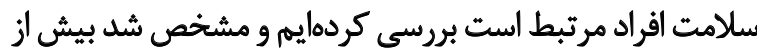

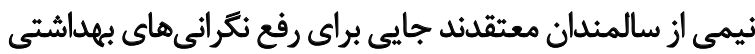

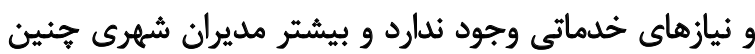

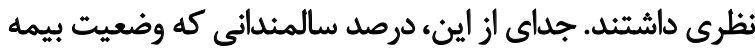
سلامت را بد مى دانستئد بيشتر از مديران بود [1A] هرجند در اين مطالعه دليل اين ديدكاه سالمندان توضيح داده

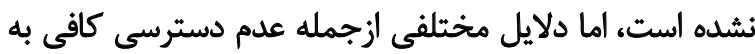

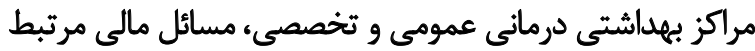

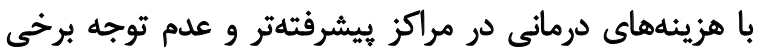

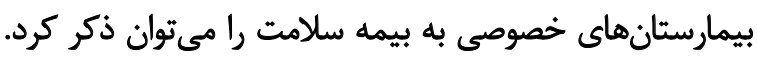

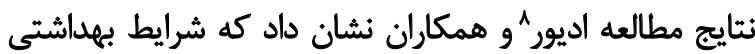

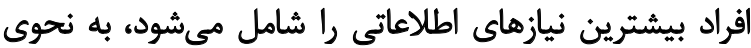

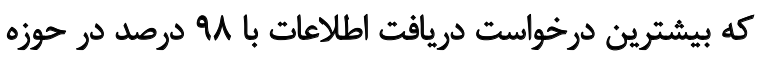

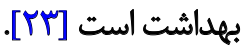
ازي جمله محدوديتهاي اين مطالعه، عدم همكارى كافي در

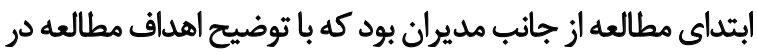

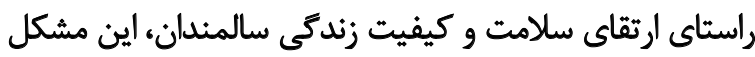
برطرف شد. محدوديت ديكر اين مطالعه عدم تعميميذئيرى نتايج سايج

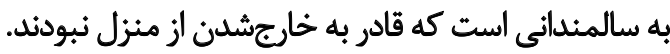

مالاحظاث اخلاقي بيروى أز أصول اخلاق يُوهش يُؤوش حاضر در كميته اخلاق دانشكاه علوميزشكى قزوين 


\section{References}

[1] Zarghani SH, Kharazmi OA, Johari L. Evaluation of the "AgeFriendly" city indicators in Mashhad by focusing on the socialcultural indices. Human Geography Research. 2015; 47(4):67388. [In Persian]

[2] Beard J. Network of cities tackles age-old problems. Bull World Health Organ. 2010; 88:406-7. [DOI:10.2471/BLT.10.020610]

[3] Orang Z, Mazloom Z, Hejazi N. Malnutrition and anthropometric measurements among elderly people with cardiovascular diseases. J Qazvin Univ Med Sci. 2018; 21(6):46-54. [In Persian] [DOI:10.29252/qums.21.6.54]

[4] Ravanipour M, Salehi S, Taleghani F, Abedi HA. Power in old age: A qualitative study. Iran J Psychiatr Clin Psychol. 2009; 14(4):447-53. [In Persian]

[5] Kharazmi O, Zarghani H, Johari L. Evaluation of urban open spaces and transport in the city of Mashhad on the basis of age-friendly city indices (participatory approach) . $6^{\text {th }}$ National Conference of planning and urban management, with an emphasis on the components of the Islamic city. 2014 Nov 12-13; Mashhad, Iran. [In Persian]

[6] Emlet CA, Moceri JT. The importance of social connectedness in building age-friendly communities. J Aging Res. 2012; 173247:1-9 . [DOI:10.1155/2012/173247]

[7] Lai M-M, Lein S-Y, Lau S-H, Lai M-L. Modeling Age-Friendly Environment, Active Aging, and Social Connectedness in an Emerging Asian Economy. J Aging Res. 2016; 2052380. [DOI:10.1155/2016/2052380]

[8] Newall N, Menec V. Age-Friendly Communities Principles and Initiatives. Can Acoust. 2014; 42(3):1-2.

[9] Jackisch J, Zamaro G, Green G, Huber M. Is a healthy city also an age-friendly city? Health Promot Int. 2015; 30(suppl 1):i108-i17. [DOI:10.1093/heapro/dav039]

[10] Nemati D, Aghbakhshi H. Tehran's elderly friendly city, first steps to realizing world's first elderly capital. Soc Res. 2013; 6(18):15-44. [In Persian]

[11] Nazari H, Rashedi V, Mohammadi H, Yousefi M. Relationship between cognitive status and activities of daily living among the elderly of nursing homes. J kermanshah Univ Med Sci. 2015; 18(12):744-6. [In Persian]

[12] Coleman VJ. The Greying of American cities: Evaluating built environment indicators for ensuring an age-friendly city. https://escholarshiporg/uc/ucla_etd. 2015.

[13] Buffel T, Phillipson C. Ageing in urban environments: Developing 'age-friendly'cities. Crit Soc Policy. 2012; 32(4):597-617. [DOI:10.1177/0261018311430457]

[14] Easaloo S, Khaksari Rafsanjani A, Jomeh poor M. Elderly needs and problems in urban space. Case study: (Streets of Central Qom City). Soc Res J. 2015; 2(6):1-41. [In Persian]

[15] Bastani F, Haghani H, Goodarzi P. Age-friendly cities features from the elderly's perspectives underscoring "Community Support and Health Services". J Client-Centered Nurs Care. 2016; 2(2):103-14. [In Persian] [DOI:10.32598/jccnc.2.2.103]
[16] Sharqi A, Zarghami E, Salehi Kousalari F, Olfat M. Evaluating status of global indices of age-friendly city in Tehran Metropolis (AFC). Urban Reg Stud Res. 2016; 8(28):1-22. [In Persian] [DOI:10.21307/acee-2016-034]

[17] Eslami S. Urban planning to create age-friendly city (Case Study: Ardabil City). Ardabili: University of Mohaghegh Ardabili Faculty of Human Sciences Department of Geography; 2017. [In Persian]

[18] Taraghi Z, llali ES. A comparison of older adults' and managers' attitudes towards age-friendly city indexes. Iranian Journal of Ageing. 2018; 13(2):236-49. [In Persian] [DOI:10.32598/ sija.13.2.236]

[19] Mahfoozpour S, Iranfar M, Ainy E. Determinant factors and elderly perspectives of users' friendly transportation. Saf Promot Inj Prev. 2017; 5(1):9-14. [In Persian]

[20] Fobker S, Grotz R. Everyday mobility of elderly people in different urban settings: The example of the City of Bonn, Germany. Urban Stud. 2006; 43(1):99-118. [DOI:10.1080/00420980500409292]

[21] Siu O-I, Phillips DR, Leung T-w. Age differences in safety attitudes and safety performance in Hong Kong construction workers. J Safety Res. 2003; 34(2):199-205. [DOI:10.1016/ S0022-4375(02)00072-5]

[22] Fiedler M. Older people and public transport. Challenges and changes of an ageing society final report. Available at: http:// www emta com/IMG/pdf/Final_Report_Older_People_protec pdf. 2007.

[23] Edewor N, ljiekhuamhen OP, UP. E-u. Elderly people and their information needs. Library Philosophy and Practice. (e-journal) serial on the internet Available at: http:// digitalcommonsunledu/l. 2016:16.] 